\section{Primary tuberculosis masquerading as gingival enlargement}

\author{
J. S. Gill, ${ }^{1}$ S. Sandhu' ${ }^{2}$ and S. Gill ${ }^{3}$ \\ VERIFIABLE CPD PAPER
}
IN BRIEF
- Tuberculosis is a highly infectious communicable disease and is a major cause of death worldwide.
- Primary tuberculosis manifesting in the gingiva as diffuse gingival enlargement is a rare finding.
- This article highlights the importance of oral tuberculosis diagnosis in the dental surgery: early detection, prompt treatment and appropriate infection control methods.

\begin{abstract}
Background Tuberculosis is a chronic granulomatous disease and an enormous health problem. Oral lesions are relatively uncommon, however, pulmonary tuberculosis may manifest with the involvement of the gingiva as the only primary finding. Case description The present case study reports tuberculosis of the gingiva manifesting as gingival enlargement. The diagnosis was based on sputum examination, histopathology and immunologic investigations. Anti-tubercular therapy was carried out with remarkable changes in gingival health within four months. Clinical implications and conclusion Gingival tuberculosis is a relatively uncommon occurrence and this case study emphasises the need for the clinician to include tuberculosis in the differential diagnosis of various types of gingival enlargement. Early detection and prompt treatment is the key for management of this highly infectious and communicable disease.
\end{abstract}

Tuberculosis (TB) is a chronic infectious granulomatous disease with a worldwide distribution. ${ }^{1,2}$ It remains an enormous health problem in most developing countries. ${ }^{2}$ The SouthEast Asian region carries a disproportionate $88 \%$ of the world's burden of tuberculosis. ${ }^{3}$ India alone accounts for nearly one fifth of the global burden of tuberculosis. ${ }^{1}$ Every year approximately 2.2 million develop tuberculosis in India of which around 0.87 million are infectious cases and it is estimated that annually there are around 330,000 deaths due to tuberculosis. ${ }^{1}$ Moreover, in areas of the world where HIV infection is prevalent, tuberculosis has become the most common opportunistic infection. Nearly 4 million in India are infected with HIV and approximately half of these are also infected with M. tuberculosis. ${ }^{3}$ The vulnerability towards tuberculosis in developing countries results from multiple factors including poverty, economic recession, malnutrition and cases of multidrug resistance. ${ }^{2}$

Senior Lecturer, ${ }^{2}$ Professor and /Head, Periodontology, Department of Periodontics, SGT Dental College, Hospital \& Research Institute, Budhera, Gurgaon, Haryana, India; ${ }^{3}$ Residency student, Oral Pathology, Department of Oral Pathology, GDC, Ahmedabad, Gujarat. India ${ }^{*}$ Correspondence to: Jaspreet Singh Gill Email:drjaspreet13137@yahoo.com

\section{Refereed Paper}

Accepted 19 February 2010

DOI: $10.1038 /$ sj.bdj.2010.344

${ }^{\circledR}$ British Dental Journal 2010; 208: 343-346
Tuberculosis is caused by the bacteria Mycobacterium tuberculosis, Mycobacterium bovis and sometimes with atypical Mycobacteria. ${ }^{4,5}$ TB mainly affects the lungs but also affects intestines, meninges, bones, joints, lymph glands, skin and other tissues of the body. ${ }^{3}$ Oral tuberculous lesions are infrequent and it is estimated that only $0.05-5 \%$ of total tuberculosis cases may present with oral manifestations. ${ }^{6}$ The tubercular lesions of the oral mucosa are seldom primary, but are rather secondary to pulmonary disease. Primary oral tuberculosis lesions are extremely rare and usually seen in children but may also occur in adults. It typically involves the gingiva and is associated with regional lymphadenopathy. Secondary tuberculosis lesions are more frequent and involve the tongue followed by the palate, lips, buccal mucosa, gingiva and frenula. ${ }^{7-19}$ Apart from these, TB lesions have also been shown to affect the salivary glands, tonsils, uvula and alveolar mucosa. ${ }^{20}$

The oral tuberculous lesions appear as ulcers, patches, indurated soft tissue and occasionally within the jaw in the form of tuberculous osteomyelitis. ${ }^{21-26}$ A majority of the cases of oral tuberculosis often appear as chronic non-healing ulcers and so far only two cases of primary tuberculosis manifesting as gingival enlargement have been reported. ${ }^{18,19}$ In the present case study, we report an unusual and infrequent case of gingival tuberculosis manifesting as diffuse gingival enlargement.

\section{CASE REPORT}

A 32-year-old female reported to the Department of Periodontics, SGT, Dental College and Hospital, Gurgaon, Haryana, India with a chief complaint of non-painful swelling and bleeding gums, particularly on the upper right side of the mouth. The patient had a history of gingival enlargement over the past two years, gradually increasing over time. There was insignificant improvement in her gingival condition even after a dentist had performed a full mouth gingivectomy about four months previously. After aggravation of her gingival condition she eventually reported to our hospital. Her detailed medical history revealed that she had experienced regular weight loss (around $3 \mathrm{~kg}$ ) over the past four to five months and she also complained of a cough and feelings of malaise over the past 15 days. However, her family history was non-contributory and she was not on any kind of systemic medication. A comprehensive intra-oral examination showed no hard tissue abnormalities. However, examination of the periodontium revealed the involvement of the gingiva, while the rest of the lining mucosa and soft tissues were healthy. Gingival enlargement was present 
especially in the upper anterior labial and posterior buccal areas. The gingiva were fiery red, irregular, pebbled, granular and unusual in appearance (Fig. 1). It was painless to touch, but bled spontaneously on provocation. Papillary enlargement was also present in the lower mandibular gingiva and the patient had poor oral hygiene. There was no clinical attachment loss and a panoramic examination (OPG) confirmed no alveolar bone loss in the patient. Extraoral examination revealed no significant cervical lymphadenopathy. Based upon the initial clinical examination, a provisional diagnosis of inflammatory gingival enlargement was made.

The various pathologic entities included in the differential diagnosis were enlargement due to drugs, malignancies, traumatic ulcers and/or infection (bacterial, fungal and viral). Since the patient was not on any systemic medication, gingival enlargement due to drugs was ruled out. Various routine investigations were advised to investigate the possibility of this atypical appearance of the gingiva. The results of complete blood count, urine analysis and biochemical tests were within normal limits, ruling out the possibility of leukaemic associated enlargement. HIV, HBs antigen tests and VDRL (venereal disease research laboratory) were also negative, eliminating any infectious aetiology of the enlargement. However, erythrocyte sedimentation rate (ESR) was elevated. Furthermore, a complete supragingival debridement under universal precautions did not show any improvement in the overcall condition of the gingiva. Thus, an incisional biopsy was performed on the upper labial gingiva in relation to the maxillary right central incisor (11) and from the lower labial gingiva with respect to the mandibular lateral incisor (32). The histopathological examination showed hyperplasia of the stratified squamous epithelium along with parakeratosis. In the connective tissue, multiple granulomas formed by epitheloid cells, Langhans' type giant cells and lymphocytes with caseous necrosis in the centre were seen. These features were suggestive of a tuberculous granulomatous lesion (Fig. 2).

A periodic acid shiff (PAS) and grocotigomori staining ruled out the presence of fungal infection in the granulomatous areas. The patient was referred to the medical department for further investigations. Acid fast bacilli (AFB) were detected in a sputum smear. For developing countries like India, the smear microscopy is likely to remain in the foreseeable future the only cost effective tool for diagnosing patients with infectious tuberculosis and monitoring the progress of treatment. ${ }^{27}$ An ELISA test confirmed the presence of antibodies against mycobacterium tuberculosis. However, chest X-ray (CXR) did not reveal any characteristic findings, thus ruling out any pulmonary involvement. Based on all the above observations, the patient was confirmed as a case of gingival tuberculosis. WHO-recommended Directly Observed Treatment, Short Course (DOTS) strategy was launched formally as the Revised National TB Control programme in India in 1997. DOTS has been widely advocated and successfully applied and is today the international standard for TB control programmes. Thus, the physician initiated WHO recommended category 1 anti-tubercular therapy (DOTS) under the Revised National Tuberculosis control programme with rifampicin (450 mg), isoniazid (600 mg), ethambutol (1,200 mg) and pyrazinamide $(1,500 \mathrm{mg})$ for two months with three times doses per week, followed with continuation phase with isoniazid (300 mg) and thioacetazone (150 mg) for six months. ${ }^{28}$ During this period the patient was instructed to abstain from any surgical procedure within the oral cavity and was also advised regarding the communicable nature of her present condition. However, conservative periodontal therapy was performed with minimal trauma to the gingiva and after consultation with the physician.

After the completion of four months of anti-tubercular therapy, the patient reported to our department with relatively normal gingival health, but with marginal inflammation due to poor plaque control by the patient (Fig. 3). Further, various plaque control measures were reinforced and supportive periodontal treatment was rendered.

\section{DISCUSSION}

Tuberculosis is a chronic granulomatous disease that potentially affects various systems of the human body. ${ }^{3,5,28,29}$ Tuberculosis of the oral cavity is an uncommon occurrence. It is believed that intact squamous

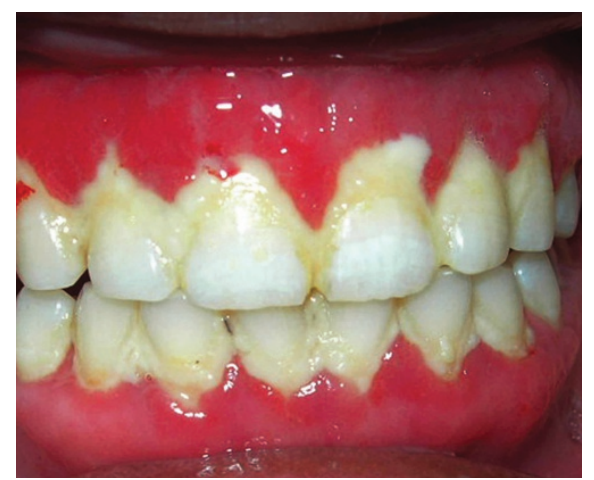

Fig. 1 The gingiva were fiery red, irregular, pebbled, granular and unusual in appearance

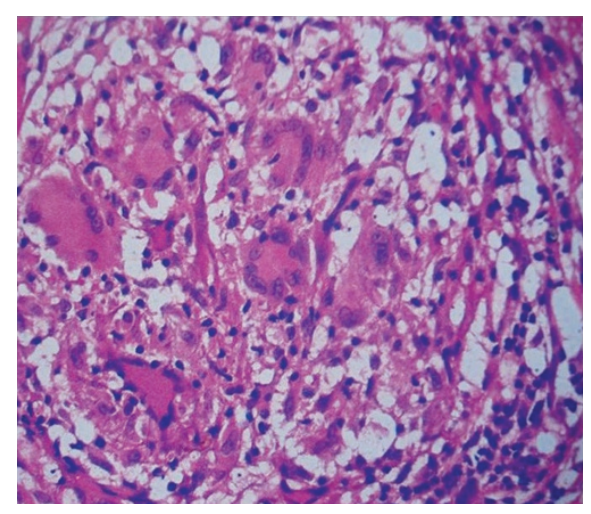

Fig. 2 Results of the histopathological examination

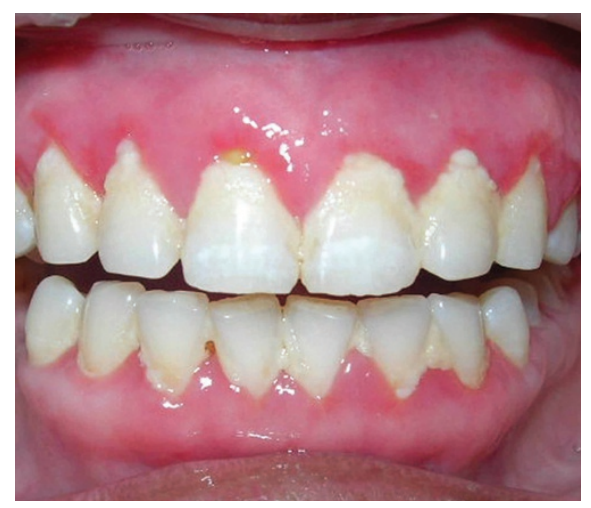

Fig. 3 The patient after the completion of four months of anti-tubercular therapy

epithelium of the oral mucosa is relatively resistant to penetration of tuberculosis bacilli and provides protection against the infection. ${ }^{30}$ The cleansing action of saliva, local $\mathrm{pH}$ and antibodies in saliva has a considerable inhibitory effect on mycobacterium.

Although the mechanism of primary inoculation has not been definitely established yet, it appears most likely that the organisms are carried in the sputum and they enter the mucosal tissue through a small break on the surface. ${ }^{3}$ Mechanical insult or tears in the mucosa caused by chronic irritation and inflammation may 
favour colonisation of the microbes .The organisms are likely to be carried to the oral tissue by a haematogenous route to be deposited in the submucosa and subsequently proliferate and ulcerate in the overlying mucosa. ${ }^{3}$ Self-inoculation with infected sputum has also been reported to play a role in the pathogenesis of primary form of tuberculosis. ${ }^{31}$ Local predisposing factors include poor hygiene, local trauma, dental extraction, leukoplakia, jaw fractures and irritation by clove oil, cyst and abscess. ${ }^{31}$ In the present case the bacteria might have spread through inflamed gingival tissues and poor oral hygiene. The living conditions and poor socioeconomic status of the patient were contributory to the unusual occurrence of the disease.

A tuberculous lesion usually appear as an irregular and well circumscribed ulcer, primary tuberculosis typically involves the gingiva and presents as diffuse, hyperaemic, nodular or papillary proliferation of gingival tissues. ${ }^{3}$ Corroborating the above findings, in the present case it appeared as diffuse gingival enlargement and the gingiva had a reddish, pebbled, erythematous appearance, which failed to respond to initial supragingival debridement. This raised suspicion and thus an incisional biopsy and other investigations were performed. The sputum examination (smear microscopy), immunological tests (ELISA) and histopathological reports confirmed the diagnosis of gingival tuberculosis. The occurrence of tuberculosis as an opportunistic infection in HIV positive cases was initially a concern. ${ }^{32}$ However, the ELISA test for HIV was negative in the patient.

Tuberculosis of the gingiva is a relatively infrequent finding and should be considered in differential diagnosis, particularly as a non-healing lesion that does not respond to usual therapy. The various pathologic entities that should be included in the differential diagnosis are enlargement due to drugs, malignancies, traumatic ulcers and/or infection (bacterial, fungal and viral). Oral tuberculosis can be a diagnostic challenge for the clinician. A complete physical examination, sputum examination, antituberculin tests, immunological investigations (antibody tests for detection of $M$. tuberculosis),,$^{33}$ scalpel biopsy (histopathological examination) and more recently molecular techniques like polymerase chain reaction (PCR) ${ }^{34}$ play a crucial role in the successful diagnosis of tuberculosis.

\section{CONCLUSION}

Although primary tuberculosis manifesting as a diffuse gingival enlargement is a rare entity, it should be included in the differential diagnosis of various types of gingival overgrowths. The clinician should be involved in the effort to control oral tuberculosis through early detection and referral of patients to a physician for proper management and by developing and implementing appropriate infection control programmes in the dental surgery.

1. World Health Organisation. Tuberculosis - the global burden. Trends in TB incidence in 9 regions of the world. 2005.

2. Chakraborty A K. Epidemiology of TB. Current status in India. Indian J Med Res 2004; 120: 248-276.

3. Bacterial infections. In Shafer W G, Hine M K, Levy B M. A textbook of oral pathology, 6th ed. Philadel phia: WB Saunders, 2009.

4. Waldman R H. Tuberculosis and the atypical mycobacteria. Otolaryngol Clin North Am 1982; 15: 581-596.

5. Phelan J A Jimenez V, Tompkins D C. Tuberculosis. Dent Clin North Am 1996; 40: 327-341.

6. Mignogna M D, Muzio L L O, Favia G et al. Oral tuberculosis: a clinical evaluation of 42 cases. Oral Dis 2000; 6: 25-30.

7. Birkholz H, Riegler H C. Tuberculosis of the tongue J Am Dent Assoc 1979; 98: 60-61.

8. Carnelio S, Rodrigues G. Primary lingual tuberculosis: a case report with review of literature. J Oral Sci 2002; 44: 55-57.

9. Gupta A, Shinde K J, Bhardwaj I. Primary lingual tuberculosis: a case report. J Laryngol Otol 1998; 112: 86-87.

10. Gupta P P, Fotedar S, Agarwal D, Sansanwal P. Primary tubercular glossitis in immunocompetent patient. Hong Kong Med J 2007; 13: 330-331.

11. Memon G A, Khushk I A. Primary tuberculosis of tongue. J Coll Physicians Surg Pak 2003; 13: 604-605.

12. Haddad N M, Zaytoun G M, Hadi U. Tuberculosis of the soft palate: an unusual presentation of oral tuberculosis. Otolaryngol Head Neck Surg 1987; 97: 91-92.

13. Kiliç A, Gül U, Gönül M, Soylu S, Cakmak S K,
Demiriz M. Orificial tuberculosis of the lip: a case report and review of the literature. Int J Dermatol 2009; 48: 178-180.

14. Rao TV, Satyanarayan CV; Sundareshwar B, Reddy C R. Unusual form of tuberculosis of lips. J Oral Surg 1977; 35: 595-596.

15. Sezer B, Zevtinoglu M, Tuncay U, Unal T. Oral mucosal ulceration: a manifestation of previously undiagnosed pulmonary tuberculosis. J Am Dent Assoc 2004; 135: 336-340.

16. Browne W G. Primary tuberculosis lesion of the oral mucosa. Br Dent J 1959; 106: 67

17. Rauch D M, Freidman E. Systemic tuberculosis initially seen as an oral ulceration: report of case. J Oral Surg 1978; 36: 387-389.

18. Karthikeyan B V, Pradeep A R, Sharma C G. Primary tuberculous gingival enlargement: rare entity. J Can Dent Assoc 2006; 72: 645-648.

19. Sharma C G, Pradeep A R, Karthikeyan B V. Primary tuberculosis clinically presenting as gingival enlargement: a case report. J Contemp Dent Pract 2006; 7: 108-114.

20. Kim $Y H$, Jeong $W J$, Jung $K Y$, Sung $M W$ et al. Diagnosis of major salivary gland tuberculosis: experience of eight cases and review of the literature Acta Otolaryngol 2005; 125: 1318-1322.

21. Dixit R, Sharma $S$, Nuwal P. Tuberculosis of oral cavity. India J Tuberc 2008; 55: 51-53.

22. Dimitrakopoulos I, Zouloumis L, Lazaridis N, Karakasis D et al. Primary tuberculosis of the oral cavity. Oral Surg Oral Med Oral Pathol 1991; 72: 712-715.

23. Ebenezer J, Samuel R, Mathew G C, Koshy S et al. Primary oral tuberculosis. Report of two cases. Indian J Dent Res 2006; 17: 41-44.

24. Koloktromis A, Antoniadis D, Trigonidis G Papanagioton P. Oral tuberculosis. Oral Dis 1996; 2: 242-243.

25. Mani N J. Tuberculosis initially diagnosed by asymptomatic oral lesions. A report of three cases. J Oral Med 1985; 40: 39-42.

26. Garber H T, Harrigan W. Tuberculosis osteomyelitis of the mandible with pathologic fracture. J Oral Surg 1978; 36: 144-146.

27. Indian Council of Medical Research (ICMR) bulletin. Indian Coun Med Res 2002; 32(8)

28. Central TB Division. Directorate General of Health Services. Ministry of Health and Family Welfare. RNTCP at a glance. Revised National TB Control Programme. Government of India. http://uttarkashi. nic.in/Dept/Health/RNTCP/RNTCP.pdf.

29. Cawson R A, Odell E W. Essentials of oral pathology and oral medicine, $6^{\text {th }}$ ed. New York: Churchill Livingstone, 1998.

30. Pekiner F N, Erseven G, Borahan M O, Gümrü B. Natural barrier in primary tuberculosis inoculation: oral mucous membrane. Int J Tuberc Lung Dis 2006: 10: 1418.

31. Rodrigues G, Carnelio S, Valliathan M. Primary isolated gingival tuberculosis. Braz J Infect Dis 2007; 11: 172-173.

32. Feller $L$, Anagnostopoulos $C$, Bouckaert $M_{1}$ Raubenheimer E J. HIV/TB co-infection: literature review and report of multiple tuberculosis oral ulcers. SADJ 2005; 60: 330-332, 343.

33. Fujibayashi T, Takashashi Y, Yoneda T, Tagami Y. Tuberculosis of tongue: a case report with immunological study. Oral Surg Oral Med Oral Pathol 1970; 47: 427-435.

34. Rivera H, Correa M F, Castillo-Castillo S, Nikitakis N G. Primary oral tuberculosis: a report of a case diagnosed by polymerase chain reaction. Oral Dis 2003; 9: 46-48. 